\title{
The Radiologic Comparison of Operative Treatment Using a Hook Plate versus a Distal Clavicle Locking Plate of Distal Clavicle Fracture
}

\author{
Byungil Yoon, Jae Yoon Kim ${ }^{\varpi}$, Jae-Sung Lee, Hyoung Seok Jung \\ Department of Orthopaedic Surgery, Chung-Ang University College of Medicine, Seoul, Korea
}

\begin{abstract}
Background: The purpose of this study was to compare the radiologic results of patients who underwent surgery with a hook plate and a locking plate in distal clavicle fractures.

Methods: Sixty patients underwent surgical treatment for Neer type Ila, Ilb, III, and V distal clavicle fracture. Twenty-eight patients underwent fracture fixation with a hook plate and 32 with a locking plate. Coracoclavicular distance was measured on standard anteroposterior radiographs before and after the surgery, and union was confirmed by radiograph or computed tomography taken at 6 months postoperatively. Other radiologic complications like osteolysis was also checked.

Results: Bony union was confirmed in 59 patients out of 60 patients, and 1 patient in the hook plate group showed delayed union. Coracoclavicular distance was decreased more in the hook plate group after surgery $(p<0.01)$. After 6 weeks of the hook plate removal, the coracoclavicular distance was increased a little compared to before metal removal, but there was no difference compared to the contralateral shoulder. Eleven out of 28 patients (39.3\%) showed osteolysis on the acromial undersurface in the hook plate group.

Conclusions: Both the hook plate group and the locking plate group showed satisfactory radiologic results in distal clavicle fractures. Both hook plate and locking plate could be a good treatment option if it is used in proper indication in distal clavicle fracture with acromioclavicular subluxation or dislocation.
\end{abstract}

(Clin Shoulder Elbow 2018;21(4):227-233)

Key Words: Clavicle; Bone plates; Open fracture reductio

\section{Introduction}

Distal clavicle fractures account for about $15 \%$ to $25 \%$ of all clavicle fractures. ${ }^{1,2}$ Many surgical techniques for unstable distal clavicle fractures requiring surgical treatment, such as fixation with Kirschner wire (K-wire), ${ }^{3)}$ Weaver-Dunn operation, ${ }^{4)}$ tension band wiring, ${ }^{3,5)}$ coracoclavicular screw fixation, ${ }^{6-8)}$ fixation with plate, ${ }^{9-12)}$ and arthroscopic coracoclavicular ligament repair using endobutton, ${ }^{13)}$ have been introduced. Among metal plate fixation techniques, the use of a hook plate facilitates reduction of superior migration of proximal bone fragments and maintenance of reduction, and makes it possible to maintain reduction indirectly without direct fixation of distal fragments with screws when distal fragments are very small. ${ }^{14)}$ However, subacromial osteolysis is more likely to occur in the subacromial space where the hook plate is placed, ${ }^{15,16)}$ and it is impossible to recover the full range of motion (ROM) due to the hook plate. Therefore, it is known that it is better to remove the hook plate within 6 months after surgery. However, 6 months may not be sufficient for fracture healing. ${ }^{17)}$ In addition, side effects such as rotator cuff tears, subacromial impingement syndrome, ${ }^{18)}$ and periprosthetic fractures ${ }^{19)}$ have been reported. On the other hand, fixation with a locking plate involves the difficulty of direct placement of screws on distal fragments, and has the disadvantage that it cannot be performed if distal fragments are small. ${ }^{20)}$ In addition, distal clavicle fractures accompanied by a rupture of the coraco-

Received June 15, 2018. Revised September 20, 2018. Accepted September 21, 2018.

Correspondence to: Jae Yoon Kim

Department of Orthopaedic Surgery, Chung-Ang University Hospital, 102 Heukseok-ro, Dongjak-gu, Seoul 06973, Korea

Tel: +82-2-6299-1592, Fax: +82-2-1800-1114, E-mail: kjycje@gmail.com, ORCID: https://orcid.org/0000-0001-8099-1366

IRB approval: Chung-Ang University Hospital (IRB No. 1807-005-16186).

Financial support: None. Conflict of interests: None. 
clavicular ligament have the problem that the coracoclavicular distance is not easily restored without performing direct repair or reconstruction of the coracoclavicular ligament, ${ }^{21)}$ but the abovementioned disadvantages of hook plates can be complemented or overcome. In this study, we aim to compare the radiological results of patients with distal clavicle fractures who were surgically treated with a hook plate or a locking plate.

\section{Methods}

The subjects were 60 patients who underwent internal fixation with a $3.5 \mathrm{~mm}$ LCP Clavicle Hook Plate (Synthes, West Chester, PA, USA) or Locking Clavicle Plate (Hankil Techmedical Co., Hwaseong, Korea) from 2009 to 2017 among patients with Neer type Ila, IIb, III, and V distal clavicle fractures. The patients who had Neer type I or IV distal clavicle fractures, received conservative treatment, underwent fixation with only K-wires or screws, or underwent coracoclavicular ligament reconstruction were excluded from this study. Among the subjects, 28 patients underwent surgery with a hook plate, and 32 patients with a locking plate. Metal removal was performed when we were able to detect signs of fracture healing on plain radiographs or computed tomography images (CTs) taken at 6 months after surgery. Since complications such as osteolysis or fracture may occur in patients who underwent surgery with a hook metal plate, we planned to perform implant removal if fracture union was detected 6 months after surgery, and locking plate removal was performed within one year after surgery at the latest. At 6 weeks after implant removal, clavicular anteroposterior plain radiographs were taken, the coracoclavicular distance of both the left and right sides was measured. In addition, when a hook plate was used, it was also examined whether osteolysis around the hook or other complications like a fracture occurred.

\section{Surgical Methods and Postoperative Management}

Under general anesthesia, the patient was placed in the beach chair position and a skin incision was made over the distal clavicle fracture site using the standard approach. After exposing the fracture site and performing reduction, fixation was performed with a locking plate when it was possible to place at least three screws in the distal fragment, but when they were small or the fracture was severely comminuted, distal fragments were fixed with a hook plate (Fig. 1, 2). After confirming that satisfactory reduction was achieved using C-arm fluoroscopy, fixation was performed using a metal plate and screws. Additional reduction and fixation for displaced bone fragments were performed with a K-wire or wire loop if necessary (Fig. 3). K-wires were additionally used in 1 case of the hook plate group and in 4 cases of the locking plate group, and wire loops were additionally used in 1 case of the hook plate group. No patient in either of the two groups underwent coracoclavicular ligament repair. All the patients wore a shoulder immobilizer for 6 weeks postoperatively, and active ROM exercises of the elbow joint, wrist joint, and fingers were performed immediately after surgery. At 6 weeks postoperatively, the shoulder immobilizer was removed and active ROM exercises of the shoulder joint were performed.
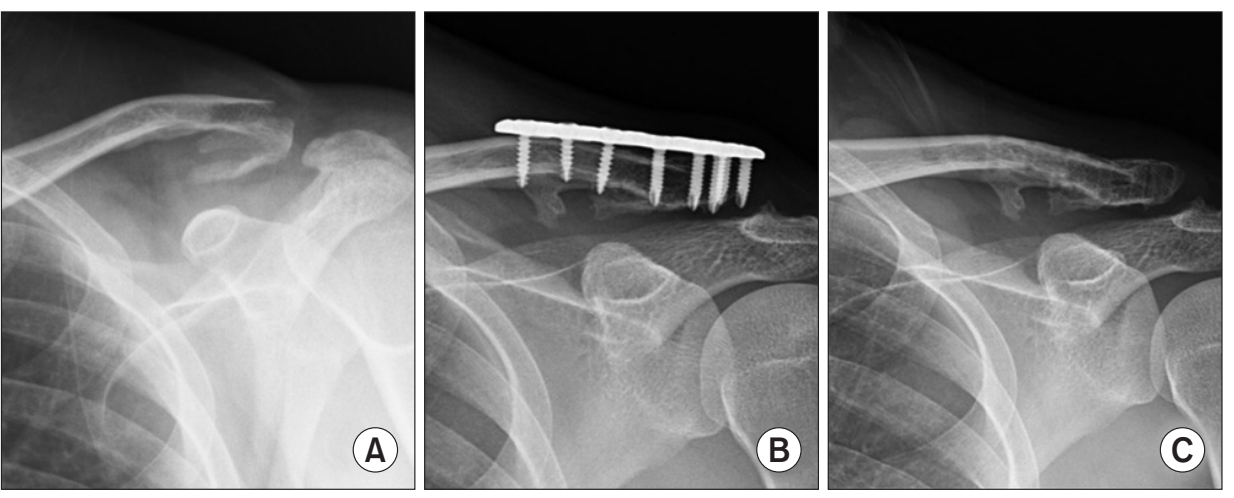

Fig. 1. (A) A 30-year-old male sustained a Neer type IIa left distal clavicle fracture. (B) The fracture was reduced with locking plate. (C) The last follow-up radiograph after implant removal shows solid union.
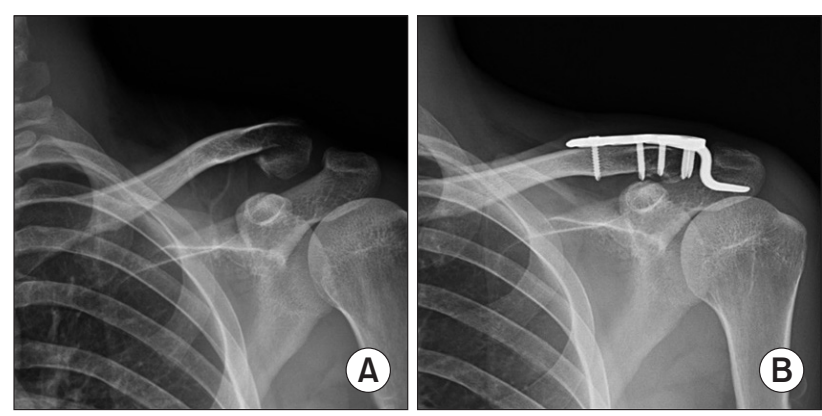

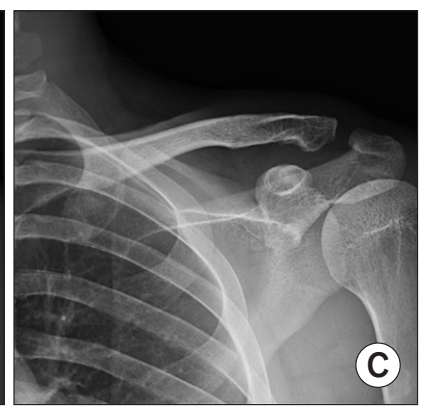

Fig. 2. (A) A 27-year-old male sustained a Neer type IIb left distal clavicle fracture. (B) The fracture was reduced and fixed with hook plate. (C) The last follow-up radiograph after implant removal shows solid union. 


\section{Statistical Analysis}

Statistical analysis was performed using IBM SPSS ver. 22 (IBM Co., Armonk, NY, USA). The independent t-test and chi-square test were performed, the significance level was defined as 0.05 , and if the $p$-value was less than 0.05 , it was considered to indicate a statistically significant difference.

\section{Results}

There was no statistical difference in demographic data
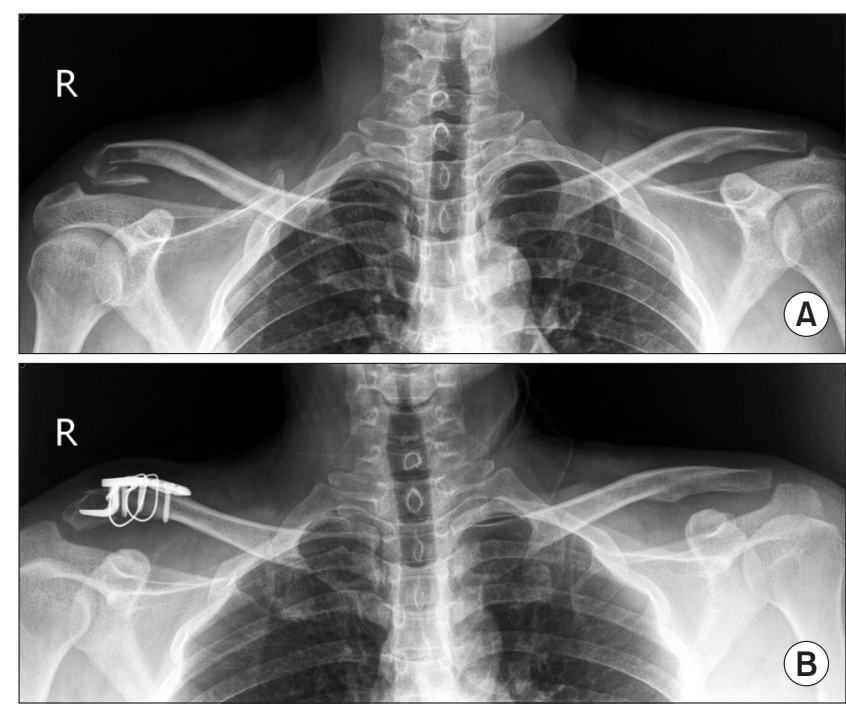

Fig. 3. (A) A 37-year-old male sustained a Neer type V right distal clavicle fracture with acromioclavicular joint subluxation. (B) The fracture-dislocation was reduced and fixed with hook plate and wiring. between the two groups (Table 1). A total of 60 cases of distal clavicle fractures included 23 cases of Neer type Ila, 18 cases of Neer type IIb, 1 case of Neer type III, and 18 cases of Neer type V. There was no difference in fracture types classified according to the Neer classification between the two groups $(p=0.26)$ (Table 2). There were no specific complications after surgical treatment in all of 60 patients. The mean operative time was $65.7 \pm 14.8$ minutes in the hook plate group and $70.5 \pm 17.2$ minutes in the plate group. There was no significant difference in operative time between the two groups $(p=0.25)$. Complete fracture

Table 1. Patient Demographic Data

\begin{tabular}{lccc}
\hline \multicolumn{1}{c}{ Variable } & Hook plate & Locking plate & $p$-value \\
\hline No. of patient & $28(100)$ & $32(100)$ & \\
Age (yr) & $39.9 \pm 14.8$ & $47.4 \pm 14.8$ & 0.054 \\
Sex (male/female) & $24(85.7) / 4(14.3)$ & $21(65.6) / 11(34.4)$ & 0.073 \\
Fracture site (right/left) & $13(46.4) / 15(53.6)$ & $9(28.1) / 23(71.9)$ & 0.142 \\
\hline
\end{tabular}

Values are presented as number (\%) or mean \pm standard deviation.

Table 2. Neer Classification between Hook Plate and Locking Plate

\begin{tabular}{cccc}
\hline Neer classification & Hook plate & Locking plate & Total \\
\hline IIa & $8(28.6)$ & $15(46.9)$ & $23(38.3)$ \\
IIb & $8(28.6)$ & $10(31.3)$ & $18(30.0)$ \\
III & $1(3.6)$ & $0(0)$ & $1(1.7)$ \\
V & $11(39.3)$ & $7(21.9)$ & $18(30.0)$ \\
\hline
\end{tabular}

Values are presented as number (\%).
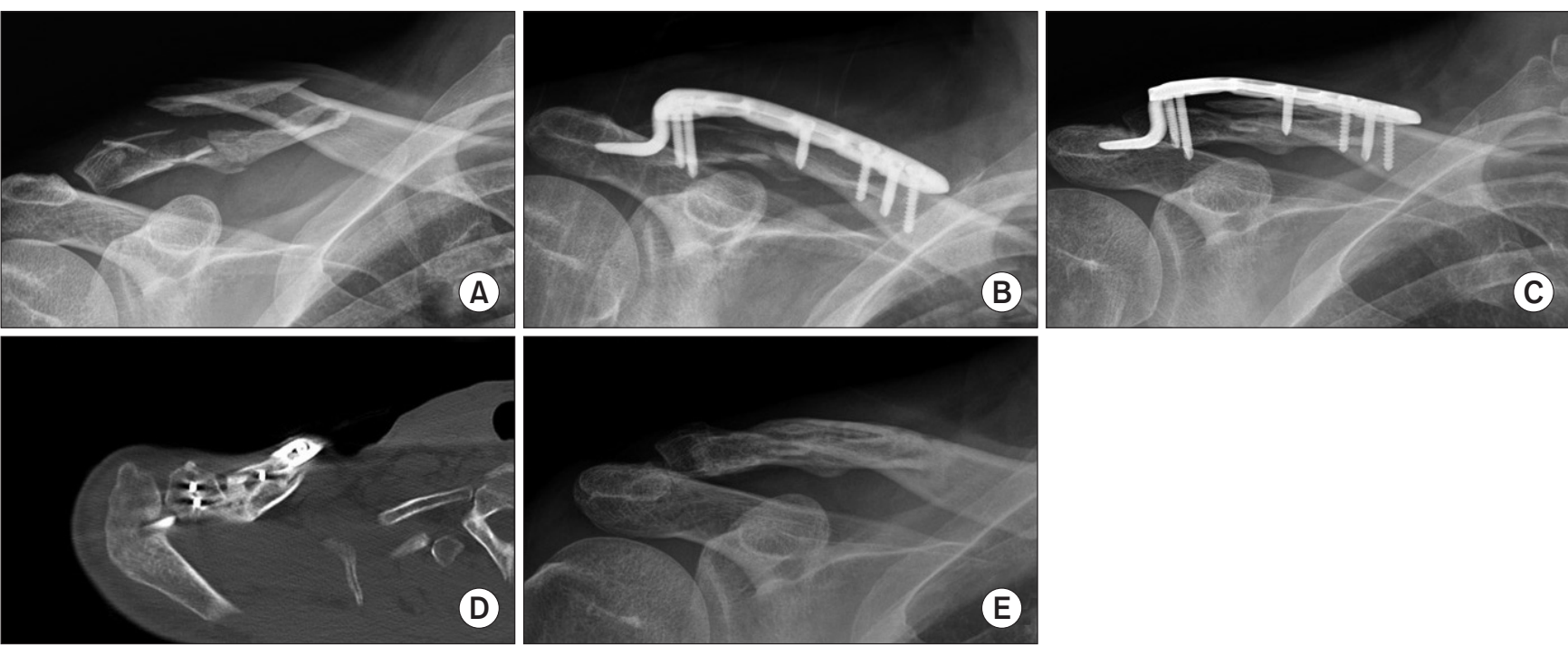

Fig. 4. (A) A 52-year-old male sustained a Neer type V right distal clavicle fracture. (B) Immediate postoperative radiograph shows fracture site reduced with hook plate. (C) Hook was moving toward to acromion on follow-up radiograph taken at 6 months postoperatively. (D) Partial union was observed on computcom tomography image taken at 6 months postoperatively. (E) Pelvic bone graft was done on nonunion site during implant removal and union was found on 3 months later. 

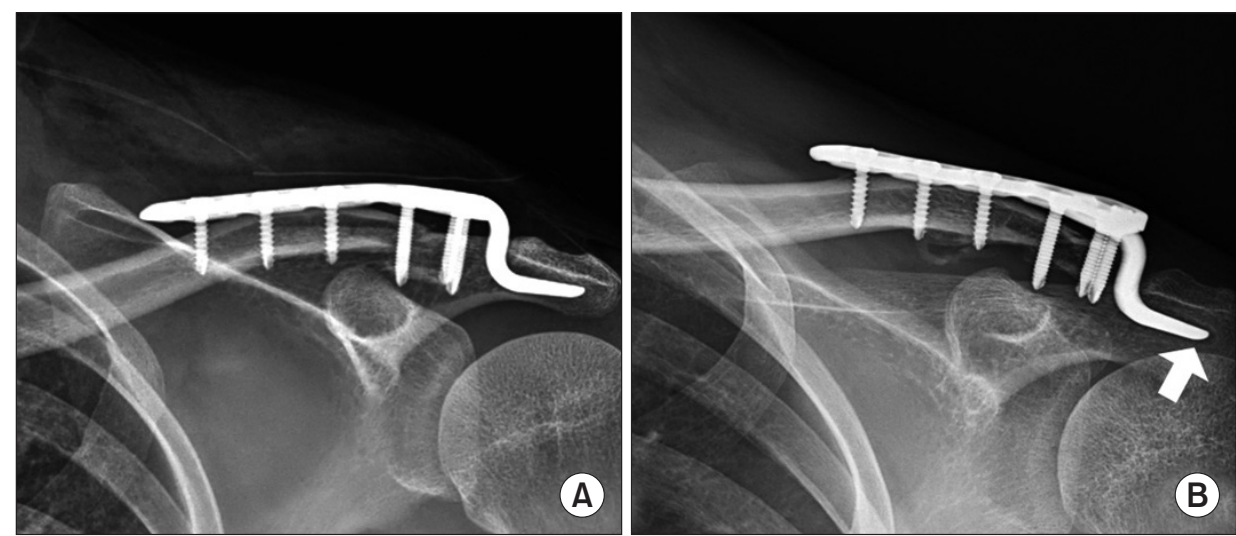

Fig. 5. (A) The immediate postoperative radiograph of a 30 -year-old male sustained a Neer type $V$ left distal clavicle fracture reduced with hook plate. (B) The last followup radiograph before implant removal shows osteolysis on acromion (white arrow).

Table 3. Overall Radiologic Outcomes (Unit: mm)

\begin{tabular}{|cccc}
\hline Variable & Hook plate & Locking plate & $p$-value \\
\hline Preoperative & & & \\
CCD & $19.4 \pm 4.3$ & $17.7 \pm 6.7$ & 0.24 \\
\hline CCD $\Delta$ & $10.3 \pm 4.3$ & $9.4 \pm 6.2$ & 0.51 \\
\hline Postoperative & & & \\
\hline CCD & $6.8 \pm 3.3$ & $10.8 \pm 2.5$ & $<0.01$ \\
CCD $\Delta$ & $-1.9 \pm 3.4$ & $3.0 \pm 2.5$ & $<0.01$ \\
\hline Postremoval & $8.2 \pm 2.6$ & $10.0 \pm 2.7$ & 0.03 \\
CCD & $-0.3 \pm 2.5$ & $1.5 \pm 2.2$ & 0.01 \\
\hline CCD $\Delta$ & & & \\
\hline
\end{tabular}

Values are presented as mean \pm standard deviation.

CCD: coracoclavicular distance of injured side as measured in anteroposterior (AP) radiographs, CCD $\Delta$ : subtraction of coracoclavicular distance of uninjured side from that of injured side as measured in bilateral AP radiographs.

healing was obtained in 59 out of 60 patients. One patient who underwent surgery with a hook plate showed delayed union at 6 months postoperatively. In the patient showing delayed union, $X$-rays taken immediately after surgery showed that the hook plate was properly placed under the acromion (Fig. 4B), but the hook gradually migrated into the acromion during the follow-up period, so we decided to remove the metal plate 6 months after surgery. Although X-ray taken at 6 months postoperatively did not show fracture union, partial bone union was detected on $\mathrm{CT}$ (Fig. 4D), so it was considered unnecessary to perform refixation. Therefore, while removing the metal plate, only autogenous iliac bone graft was performed for the site showing delayed union. $X$-ray taken at 3 months after the surgery provided radiographic findings of complete fracture union (Fig. 4E). In 11 of 28 patients (39.3\%) who were surgically treated with a hook plate, osteolysis of the acromion where the hook plate was located were detected, but there were no complications such as fractures (Fig. 5).

The mean coracoclavicular distance was measured to be 19.4 $\pm 4.3 \mathrm{~mm}$ in the hook plate group and $17.7 \pm 6.7 \mathrm{~mm}$ in the locking plate group before surgery. After surgery, the mean cora-
Table 4. Radiologic Outcome of Hook Plate in Postoperative and Postremoval (Unit: $\mathrm{mm}$ )

\begin{tabular}{cccc}
\hline Variable & Postoperative & Postremoval & $p$-value \\
\hline CCD & $6.83 \pm 3.51$ & $8.22 \pm 2.62$ & 0.022 \\
CCD $\Delta$ & $-1.74 \pm 3.65$ & $-0.30 \pm 2.48$ & 0.011 \\
\hline
\end{tabular}

Values are presented as mean \pm standard deviation.

CCD: coracoclavicular distance of injured side as measured in anteroposterior (AP) radiographs, CCD $\triangle$ : subtraction of coracoclavicular distance of uninjured side from that of injured side as measured in bilateral AP radiographs.

coclavicular distance was $6.8 \pm 3.3 \mathrm{~mm}$ in the hook plate group, and $10.8 \pm 2.5 \mathrm{~mm}$ in the locking plate group, respectively, so significant decreases were observed compared to the preoperative measures $(p<0.01)$. As for the value obtained by subtracting the coracoclavicular distance of the uninjured side from that of the injured side (henceforth, CCD $\Delta$ ), the preoperative mean value was $10.3 \pm 4.3 \mathrm{~mm}$ in the hook plate group and $9.4 \pm 6.2$ $\mathrm{mm}$ in the locking plate group, so there was no statistically significant difference between the two groups $(p=0.51)$. After surgery, the mean CCD $\Delta$ values were $-1.9 \pm 3.4 \mathrm{~mm}$ in the hook plate group and $3.0 \pm 2.5 \mathrm{~mm}$ in the locking plate group, showing that an overcorrection was made in the hook plate group compared to the degree of reduction in the locking plate group $(p<0.01)$, and that there was a significant decrease in both the groups compared to the preoperative values (Table 3).

Twenty-six out of 32 patients in the hook plate group underwent implant removal at 7.7 months (4.6 to 14.1 months) after surgery on average. In the locking plate group, 23 out of 28 patients underwent implant removal at 13.5 months (8.8 to 35.9 months) after surgery on average. In the locking plate group, there was almost no change in the coracoclavicular distance before and after implant removal, but in the hook plate group, X-ray taken at 6 weeks after implant removal showed that the coracoclavicular distance was slightly increased to $8.22 \pm 2.62$ $\mathrm{mm}$ compared to the values before implant removal, but CCD $\Delta$ was measured to be $-0.30 \pm 2.48 \mathrm{~mm}$, showing almost no difference in the coracoclavicular distance between the injured side and the contralateral side (Table 4). 


\section{Discussion}

In this study, we could obtain fracture union without major complications in both the group surgically treated with a hook plate and the group surgically treated with a locking plate for distal clavicle fractures. There was no difference in operative time between the two groups, and there was a significant decrease in the coracoclavicular distance, which had been increased preoperatively in both the groups.

Unlike hook plates, locking plates cannot fix the acromioclavicular joint, but in most cases of distal clavicle fractures, there is no rupture of the acromioclavicular ligament and the coracoclavicular ligament is attached to the distal bone fragment, so the coracoclavicular distance is expected to be improved only by fracture reduction. ${ }^{22)}$

In this study, we were also able to confirm the reduction of the coracoclavicular distance in all patients who underwent surgery with a locking plate. However, the coracoclavicular distance was different by about $3.0 \mathrm{~mm}$ from that of the uninjured side. This finding suggests that coracoclavicular distance cannot be maintained if only fracture reduction is performed without stabilizing the coracoclavicular ligament. In the hook plate group, it was found that a slight overcorrection of thecoracoclavicular distance was made compared to the uninjured side. This can be attributed to the fact that an overcorrection was attempted intentionally to avoid the loss of reduction after implant removal. In the case of acromioclavicular joint dislocations where both the acromioclavicular ligament and coracoclavicular ligament are ruptured, a partial loss of reduction is commonly observed after implant removal when fixation is performed using a hook plate. ${ }^{23)}$ However, in this study, it was found that reduction of the coracoclavicular ligament was maintained even when the hook plate was removed. This is presumed to be due to the fact that the acromioclavicular ligament is intact and the coracoclavicular ligament is partially attached to the bone fragments in most cases of distal clavicle fractures.

It is known that there is no significant difference in the clinical results of distal clavicle fractures between the cases where the difference in the coracoclavicular distance between the injured and uninjured sides is $10 \%$ or higher and the cases where the difference is less than $10 \%,{ }^{21)}$ so implications of the difference in the coracoclavicular distance are not clear yet. However, it is thought that there is a need for continuous follow-up since there has not been research on complications requiring long-term follow up such as acromioclavicular arthritis.

When a hook plate is used, it is impossible to maintain fixation for a long time due to problems such as acromion fractures ${ }^{24,25)}$ acromion osteolysis caused by the hook plate, ${ }^{26)}$ and a reduction of the ROM of the joint, ${ }^{27}$ so there are concerns about whether it is possible to maintain fixation for sufficient time until fracture union is achieved. In this study, complete fracture healing was achieved at about 6 months after surgery in all the subjects except one out of 28 patients who underwent surgical treatment with a hook plate. In one patient, delayed union was detected, so implant removal was performed. Then, the patient also showed complete bone union by autogenous bone graft alone without additional fixation. As shown in Fig. 4, $\mathrm{CT}$ taken at 6 months after surgery showed the migration of the hook of the hook plate into the acromion, so we determined that removal of the metal plate was necessary because of the possibility of fracture despite of partial union. In this case, it was expected to be difficult to obtain fracture union easily because of severe fragmentation and displacement of the fracture before surgery. Therefore, it is difficult to attribute partial union at 6 months after surgery to the use of the hook plate. In distal clavicle fractures, fixation for 6 months is thought to be enough time to obtain fracture union, and when surgery is performed with a hook plate, there is no need to make much subperiosteal dissection since the use of a hook plate obviates the need to make an effort to obtain anatomical reduction unlike when a locking plate is used. Therefore, the use of hook plates makes it possible to perform fixation minimizing damage of the blood flow to the cortical bone. In this respect, the use of hook plates may be helpful for fracture union.

Erdle et al. ${ }^{23)}$ reported that the degree of correction of the coracoclavicular distance was greater when a hook plate was used than when a locking plate was used, but that the coracoclavicular distance was larger compared to that of the uninjured side. On the other hand, in this study, the coracoclavicular distance was measured to be smaller than that of the uninjured side in the hook plate group. This is a result reflecting the intentional attempt to make a slight overcorrection compared to the uninjured side taking into account the fact that the coracoclavicular distance may be increased after implant removal. As a result, the coracoclavicular distance was measured to be smaller than that of the uninjured side while the hook plate was placed in the fracture site. However, after the hook plate was removed, the coracoclavicular distance recovered to almost the same level as the uninjured side.

In this study, we detected indications of osteolysis at the location of the hook plate in about $40 \%$ (11 out of 28 cases) of the patients treated with a hook plate, and it was found that the hook plate migrated into the acromion in 2 cases. The incidence of osteolysis was not higher compared to other studies, ${ }^{18,26,28)}$ and no major complications such as a fracture due to osteolysis or a fracture at the internal side of the hook plate occurred. ${ }^{24,25)}$ This is thought to be a result from the fact that the metal plates were removed at a relatively early stage rather than placing them in the body for a long time.

This study has several limitations which need to be pointed out. First, this study is a retrospective study. It is considered difficult to conduct a prospective study because the choice of metal 
plate fixation techniques depends on the types of fracture even though the fracture site is identical. Second, types of metal fixation devices were not randomly assigned. As mentioned above regarding the first limitation, in this study, different methods of metal plate fixation were used depending on the type of fracture. When the length of the distal fragment was enough to insert three or more screws, a locking screw was inserted to perform anatomical reduction. On the other hand, when the length of the distal fragment was not sufficient, fixation was performed with a hook plate without making much subperiosteal dissection. It is believed to be an inevitable choice due to the features of each metal fixation device, and it is thought that this factor did not have a significant impact on the results of this study because the choice of metal fixation devices was not made based on the coracoclavicular distance. Third, we made no comparison of clinical outcomes. However, both of the methods are known to show relatively good clinical outcomes, ${ }^{14)}$ and this study focused on the comparison of radiological results between the two surgical techniques because we achieved fracture union finally in all the subjects without any particular complications.

\section{Conclusion}

As a result of evaluation of the radiological results of surgical treatment of distal clavicle fractures accompanied by displacement, satisfactory fracture reduction and bone union were confirmed in both the hook plate group and the locking plate group. The coracoclavicular distance was more decreased in the hook plate group than in the locking plate group, and fracture reduction was also more stably maintained in the hook plate group after the metal plate was removed. In many cases, radiographs showed phenomena such as osteolysis, but there were no radiological findings of problems such as fractures when implant removal was performed approximately 6 months after surgery.

\section{References}

1. Edwards DJ, Kavanagh T, Flannery MC. Fractures of the distal clavicle: a case for fixation. Injury. 1992;23(1):44-6.

2. van der Meijden OA, Gaskill TR, Millett PJ. Treatment of clavicle fractures: current concepts review. J Shoulder Elbow Surg. 2012;21(3):423-9.

3. Kao FC, Chao EK, Chen CH, Yu SW, Chen CY, Yen CY. Treatment of distal clavicle fracture using Kirschner wires and tension-band wires. J Trauma. 2001;51(3):522-5.

4. Anderson K. Evaluation and treatment of distal clavicle fractures. Clin Sports Med. 2003;22(2):319-26, vii.

5. Badhe SP, Lawrence TM, Clark DI. Tension band suturing for the treatment of displaced type 2 lateral end clavicle fractures. Arch Orthop Trauma Surg. 2007;127(1):25-8.

6. Jin $\mathrm{CZ}$, Kim HK, Min BH. Surgical treatment for distal clavicle fracture associated with coracoclavicular ligament rupture using a cannulated screw fixation technique. J Trauma. 2006; 60(6):1358-61.

7. Kwon DG, Lee TJ, Moon KH, Shin BK, Woo MS. Coracoclavicular screw fixation and tension band wiring in treatment of distal clavicle fracture. J Korean Fract Soc. 2013;26(1):1-7.

8. Macheras G, Kateros KT, Savvidou OD, Sofianos J, Fawzy EA, Papagelopoulos PJ. Coracoclavicular screw fixation for unstable distal clavicle fractures. Orthopedics. 2005;28(7):693-6.

9. Kalamaras M, Cutbush K, Robinson M. A method for internal fixation of unstable distal clavicle fractures: early observations using a new technique. J Shoulder Elbow Surg. 2008;17(1):602.

10. Kashii M, Inui H, Yamamoto K. Surgical treatment of distal clavicle fractures using the clavicular hook plate. Clin Orthop Relat Res. 2006;447:158-64.

11. Muramatsu K, Shigetomi M, Matsunaga T, Murata Y, Taguchi T. Use of the AO hook-plate for treatment of unstable fractures of the distal clavicle. Arch Orthop Trauma Surg. 2007;127(3): 191-4.

12. Tambe AD, Motkur P, Qamar A, Drew S, Turner SM. Fractures of the distal third of the clavicle treated by hook plating. Int Orthop. 2006;30(1):7-10.

13. Pujol N, Desmoineaux P, Boisrenoult P, Beaufils P. Arthroscopic treatment of comminuted distal clavicle fractures (latarjet fractures) using 2 double-button devices. Arthrosc Tech. 2013; 2(1):e61-3.

14. Zhang C, Huang J, Luo Y, Sun H. Comparison of the efficacy of a distal clavicular locking plate versus a clavicular hook plate in the treatment of unstable distal clavicle fractures and a systematic literature review. Int Orthop. 2014;38(7):1461-8.

15. Chiang CL, Yang SW, Tsai MY, Chen CKH. Acromion osteolysis and fracture after hook plate fixation for acromioclavicular joint dislocation: a case report. J Shoulder Elbow Surg. 2010;19(4):e13-5.

16. Hoffler CE, Karas SG. Transacromial erosion of a locked subacromial hook plate: case report and review of literature. J Shoulder Elbow Surg. 2010;19(3):e12-5.

17. Wolter D, Eggers C. Reposition and fixation of acromioclavicular luxation using a hooked plate. Hefte Unfallheilkd. 1984;170:80-6.

18. Lin HY, Wong PK, Ho WP, Chuang TY, Liao YS, Wong CC. Clavicular hook plate may induce subacromial shoulder impingement and rotator cuff lesion-dynamic sonographic evaluation. J Orthop Surg Res. 2014;9(1):6.

19. Charity RM, Haidar SG, Ghosh S, Tillu AB. Fixation failure of the clavicular hook plate: a report of three cases. J Orthop Surg (Hong Kong). 2006;14(3):333-5.

20. Chae SB, Choi CH, Kim DY. Usefulness of the additional Kwire fixation and suture for reinforce the treatment of distal clavicle fracture using modified tension band wiring. J Korean 
Fract Soc. 2016;29(2):107-13.

21. Shin SJ, Ko YW, Lee J, Park MG. Use of plate fixation without coracoclavicular ligament augmentation for unstable distal clavicle fractures. J Shoulder Elbow Surg. 2016;25(6):942-8.

22. Lee SK, Lee JW, Song DG, Choy WS. Precontoured locking plate fixation for displaced lateral clavicle fractures. Orthopedics. 2013;36(6):801-7.

23. Erdle B, Izadpanah $\mathrm{K}$, Jaeger $\mathrm{M}$, et al. Comparative analysis of locking plate versus hook plate osteosynthesis of Neer type IIB lateral clavicle fractures. Arch Orthop Trauma Surg. 2017; 137(5):651-62.

24. Ding M, Ni J, Hu J, Song D. Rare complication of clavicular hook plate: clavicle fracture at the medial end of the plate. J Shoulder Elbow Surg. 2011;20(7):e18-20.
25. Sohn HS, Jo BC. Results of hook plate fixation of unstable distal clavicle fractures. J Korean Fract Soc. 2011;24(4):335-40.

26. Şükür E, Öztürkmen Y, Akman YE, Güngör M. Clinical and radiological results on the fixation of Neer type 2 distal clavicle fractures with a hook plate. Acta Orthop Traumatol Turc. 2016;50(5):489-93.

27. Tan HL, Zhao JK, Qian C, Shi Y, Zhou Q. Clinical results of treatment using a clavicular hook plate versus a T-plate in Neer type II distal clavicle fractures. Orthopedics. 2012;35(8): e1191-7.

28. Chen $\mathrm{CH}$, Dong QR, Zhou RK, Zhen HQ, Jiao YJ. Effects of hook plate on shoulder function after treatment of acromioclavicular joint dislocation. Int J Clin Exp Med. 2014;7(9):256470. 